\section{L'AUSCULTATION DES MOUVEMENTS DU SOL ET DU SOUS-SOL INTERPRETATION DES MESURES}

Le terme «auscultation géotechnique " signifie l'observation, la mesure, l'analyse et la prévision du comportement d'un site naturel ou d'un ouvrage et du massif sur lequel il est fondé.

En simplifiant, on peut distinguer deux préoccupations dans l'auscultation.

La première consiste à suivre le comportement normal de l'ouvrage et du site. Cela permet, entre autres, aux ingénieurs de vérifier la validité de leurs hypothèses et de leurs calculs.

La seconde consiste à déceler aussi rapidement que possible tout phénomène accidentel ou évolutif dont la connaissance permet de juger de la santé de l'ouvrage et du site et éventuellement d'en adapter la gestion.

On se limitera dans cet article à l'auscultation des déplacements. Après une description sommaire des moyens de mesure disponibles, l'attention est portée sur l'interprétation des mesures, systématique et immédiate, et les deux préoccupations de l'auscultation sont illustrées par des exemples.

\section{SOIL DISPLACEMENTS MONITORING}

\section{INTERPRETATION OF THE MEASUREMENTS}

The expression "geotechnical testing" covers inspection, measurement, processing results and forecasting the behaviour of a natural site or of an engineering structure and the earth mass on which it is built.

A simplified analysis of this activity underlines two basic concerns.

The first consists of continuous inspection of the normal behaviour of the structure and the site. This enables the engineers to check the validity of their assumptions and of their design.

The second consists of detecting as quickly as possible any accidental or developing phenomena, the presence of which gives an indication of the soundness of the structure and the site and if need be adapting working operations.

This article deals only with monitoring of displacements. After a short summary of measurement means available, the author discusses systematic and immediate interpretation of measurements. The basic concerns of testing are illustrated by examples. 


\section{l'auscultation des mouvements du sol ou du sous-sol interprétation des mesures}

par C. LOUIS et M. DESURMONT

\section{POSITION DU PROBLEME}

Les phénomènes géologiques naturels, tels que mouvements tectoniques, érosion, dissolution, etc., ainsi que de nombreuses entreprises humaines (excavations, grands terrassements, etc.) se traduisent par des perturbations notables de l'état d'équilibre mécanique ou hydraulique des massifs. Ces perturbations se concrétisent par le développement de déplacements, de sollicitations localisées importantes et également par une modification de réseaux d'écoulement des eaux souterraines.

Pour assurer la sécurité des personnes et des biens, le géotechnicien est amené d'une part à comprendre et à analyser les lois souvent complexes qui régissent les phénomènes mécaniques ou hydrauliques dans les sols et les roches et, d'autre part, à surveiller le comportement intime des massifs susceptibles de créer un danger.

Cette dernière approche, définie par le terme auscultation, doit prétendre à plusieurs objectifs, notamment :

- analyser le comportement réel du massif et l'influence de certains phénomènes naturels ou de certaines interventions humaines (travaux);

- vérifier les résultats d'une éventuelle étude théorique et notamment le bon dimensionnement et l'efficacité des soutènements et confortements préconisés ;

- donner la possibilité de modifier le projet, si nécessaire, au vu du comportement réel du massif;

- apporter garantie et sécurité dans les zones exposées à des risques naturels et sur les chantiers. A cet effet, tout dispositif d'auscultation doit pouvoir, si nécessaire, être utilisé comme un système d'alarme moyennant certains aménagements.

- apprécier l'influence des phénomènes naturels ou travaux sur les ouvrages environnants.
L'auscultation est née suite à la réalisation de grands ouvrages tels que les barrages. Le principe d'un contrôle systématique par mesures in situ s'est généralisé dans tous les domaines de la géotechnique en s'inspirant de l'auscultation des barrages. Cela a été le cas, tout particulièrement, pour les travaux en souterrain qui ont été le cadre, au cours de ces deux dernières décennies, d'un développement intense des mesures de contrôle in situ. Cette tendance, très marquée notamment lors de l'application de la nouvelle méthode autrichienne de construction de tunnels, s'est soldée par la mise au point de nouveaux dispositifs de mesure, nombreux et variés.

Pour être efficace et constituer ainsi un véritable outil pour le géotechnicien ou le responsable du chantier, le dispositif d'auscultation adopté doit satisfaire aux conditions suivantes:

- l'appareillage doit être simple et robuste ;

- l'auscultation doit permettre un "contrôle intégral » dans l'espace et dans le temps des massifs et ouvrages soumis à des perturbations ;

- la présence des dispositifs de mesure et les mesures elles-mêmes doivent perturber au minimum le rythme de travail sur les chantiers tout en étant permanente ;

- les mesures doivent enfin être rapides et à interprétation immédiate.

Dans cette optique, il est impératif d'insister sur le but essentiel des mesures et de préciser les décisions à prendre en cas de résultats favorables ou défavorables. A cet effet, des critères sur les amplitudes, les évolutions dans le temps, les gradients des paramètres mesurés sont à définir en relation avec les conditions géologiques, la nature et la situation de l'ouvrage, etc.

\section{MOYENS D'AUSCULTATION DES DEPLACEMENTS}

Les méthodes de mesure de déplacements le plus couramment utilisées sont les suivantes:

\section{a) Méthodes optiques par mesures topographiques}

- nivellement;

- triangulation au théodolite classique ou à l'aide d'instruments électro-optiques (du type telluromètre par exemple). b) Méthodes mécaniques utilisant des appareils posés dans le massif

- extensomètre de convergence ;

- extensomètre par fils ou tiges en sondage ;

- pendule en sondage ;

- clinomètre. 
Quelle que soit la méthode utilisée, il convient d'accorder la plus grande attention à la signification de la grandeur mesurée et notamment à son caractère absolu ou relatif. Par esprit, une méthode par nivellement ou triangulation prétend apprécier un déplacement absolu, mais cela suppose un point de référence dont la fixité est vérifiée. D'une manière générale, les points de référence doivent être d'autant plus éloignés des points de mesures que les forces en jeu sont plus grandes. Par contre, les mesures d'extensométrie et de déviation sont par nature des mesures relatives car les variations de distance ou d'angle mesurées concernent des points implantés au sein du massif en déformation. Toutefois, on s'efforce d'implanter l'un des points de référence dans une zone dont le déplacement absolu est supposé le plus faible possible. Les mesures de convergence sont toujours de déplacement relatif.

\subsection{Méthodes topographiques}

Les méthodes topographiques sont trop connues pour qu'elles soient ici l'objet d'une description, même sommaire. L'accent est simplement porté sur le fait que les instruments électro-optiques, basés sur la réflexion par des cibles d'un rayon lumineux modulé, sont actuellement en plein développement.

\subsection{Extensomètres de surface}

Ces appareils sont destinés à mesurer la distance séparant deux plots scellés dans un ouvrage. Leur principe est très simple: un ruban en invar est accroché entre les deux plots de mesure et soumis à une tension constante. La lecture de la distance est faite à l'aide d'un comparateur. Les règles de convergence généralement utilisées autorisent une précision de mesure de $0.1 \mathrm{~mm}$. La figure 1 présente deux schémas de principe des mesures de convergence en tunnel et sur le gradin d'une fosse d'exploitation minière à ciel ouvert.
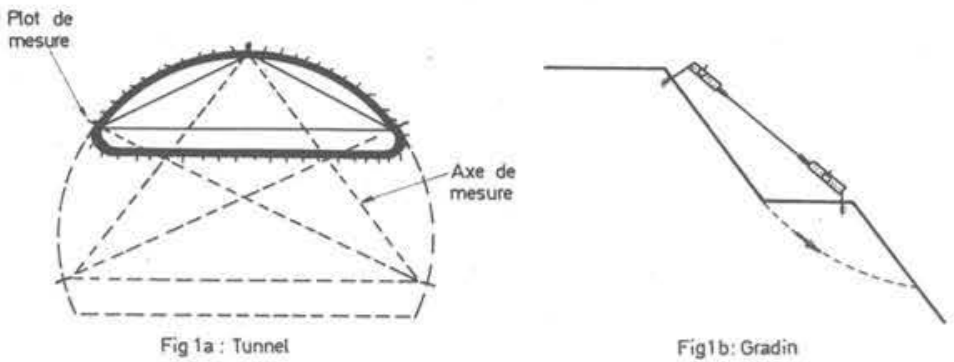

Figlb: Gradin

Fig. 1. - Schémas de principe des mesures de convergence.

\subsection{Extensomètres en sondages}

Ces appareils permettent de mesurer les déplacements relatifs d'un ou plusieurs points d'un sondage. Ils comprennent une ou plusieurs tiges rigides scellées au fond du trou et en différents points du sondage et guidées jusqu'à l'extérieur (fig. 2). L'observateur mesure à l'aide d'un comparateur les déplacements longitudinaux de l'extrémité de chaque tige sur une table de lecture scellée en tête du sondage. Il est donc possible d'évaluer avec une précision de $0.1 \mathrm{~mm}$ le déplacement relatif entre chaque point d'ancrage et la tête du sondage. Un contrôle topographique de la tête du sondage permet de déterminer les déplacements absolus de chaque point en profondeur. La mise en place

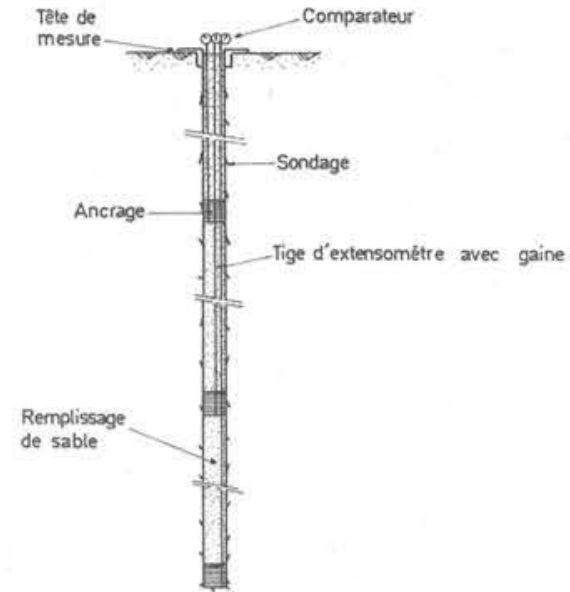

Fig. 2. - Schéma de principe d'un extensomètre triple à tiges en sondage.

d'extensomètres multiples permet de cerner la zone perturbée par les travaux.

\subsection{Pendules en sondage}

Les pendules en sondage sont basés sur le principe du fil à plomb et indiquent la verticale. On distingue les pendules directs et les pendules inverses (fig. 3).

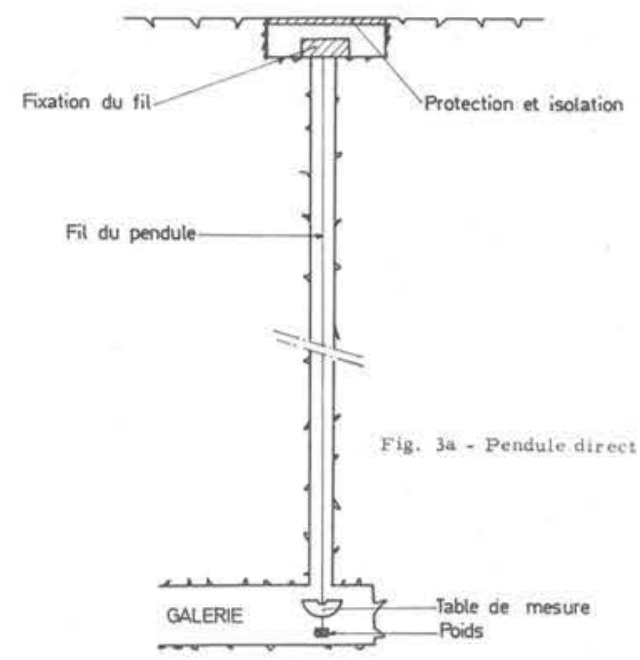

F.g. 3. - Schéma de principe des pendules direct et inverse.

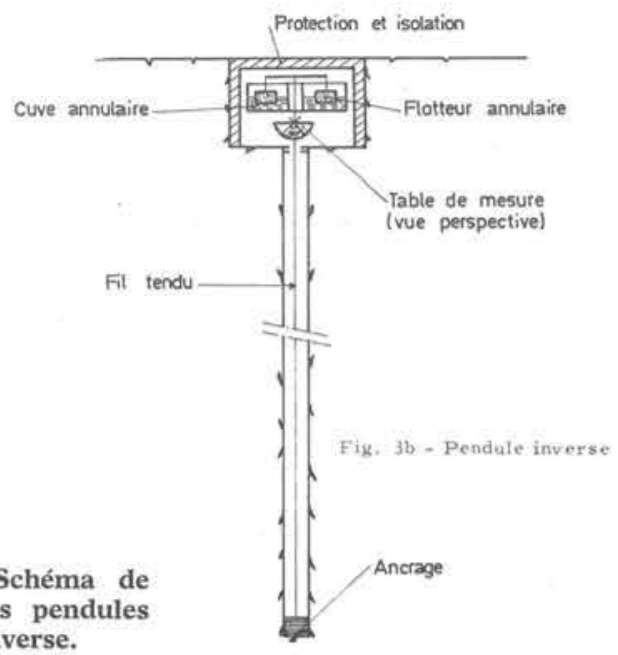


- Les pendules directs sont constitués par un fil en acier, fixé à la partie supérieure d'un trou de forage vertical. Un poids situé à l'extrémité inférieure du fil assure une tension constante du fil. A la partie inférieure, le fil tendu passe à travers une table de mesure horizontale.

- Les pendules inverses sont constitués d'un fil, ancré au fond d'un forage vertical, et dont la tension est assurée par la poussée d'Archimède exercée sur un flotteur placé à la tête du forage.

En observant les mouvements de l'extrémité libre du fil, on mesure le déplacement relatif des deux extrémités du forage.

Malgré le prix de revient et les difficultés de pose, ces appareils sont remarquables par leur exactitude (si le forage est parfaitement vertical), leur grande précision, leur sensibilité (de l'ordre de 5 microns) et leur fiabilité.

\subsection{Inclinomètres en sondage}

Le déplacement d'un sondage, perpendiculairement à son axe, peut être mesuré à l'aide d'une sonde inclinométrique (fig. 4), Cette dernière, introduite dans le sondage, circule le long d'un tubage spécial comportant des rainures. Un dispositif de mesure (pendule), équipé de jauges électriques, permet de repérer en permanence la position de l'axe du sondage.

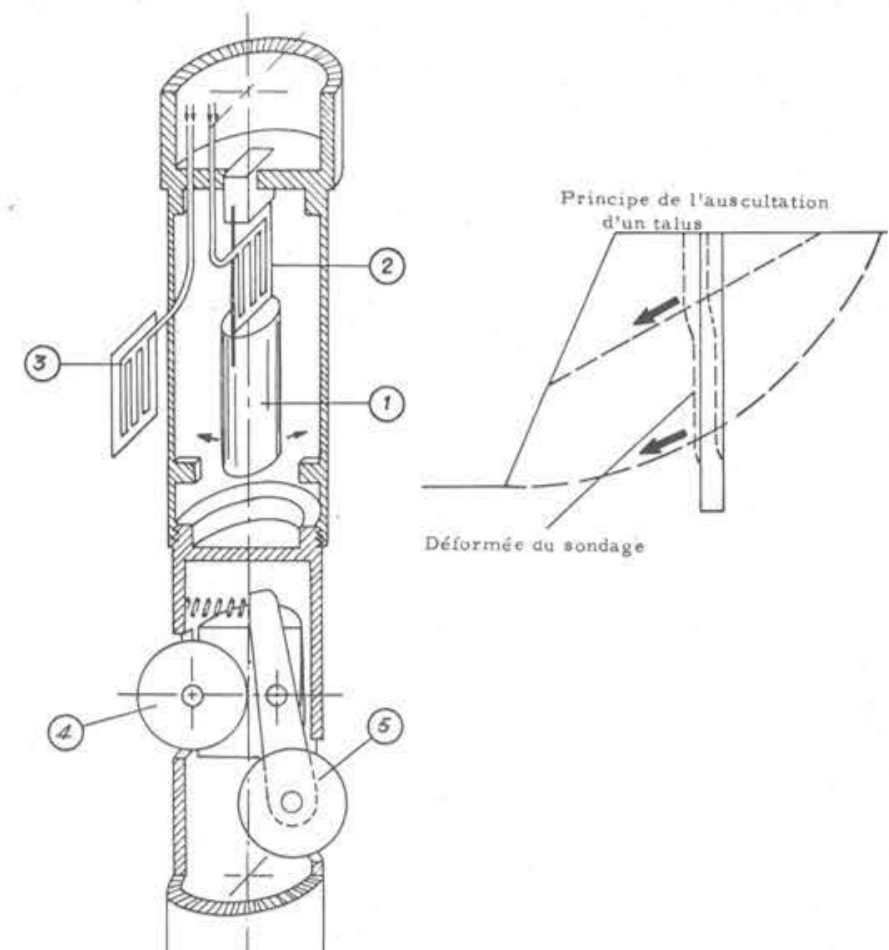

Fig. 4. - Coupe schématique partielle d'un inclinomètre à jauge de contrainte.

\section{INTERPRETATION DES MESURES DE DEPLACEMENTS}

Nous considérons ici le cas d'ouvrages souterrains dont l'auscultation des déplacements répond aux objectifs définis dans le premier paragraphe, mais aussi dont la pérénnité dans le temps doit être assurée, c'est-à-dire qu'il faut rester en-deçà de l'équilibre limite avec une "marge de sécurité acceptable». Cette catégorie d’ouvrage englobe les travaux de génie civil mais également certains travaux miniers comme, par exemple, les voies principales de desserte de travaux souterrains.

L'interprétation des mesures joue un rôle capital car elle conditionne notamment la conduite des travaux de réalisation de l'ouvrage.

Elle peut être fondée sur l'observation des quatre paramètres suivants :

- l'amplitude absolue des déplacements ;

- la vitesse des déplacements ;

- l'accélération des déplacements ;

- le taux de décroissance des déplacements ou des déformations autour de la cavité. Ce paramètre, ou gradient des déplacements ou des déformations donne une idée de l'importance de la zone d'influence du creusement de la cavité. Il conditionne pour une grande part la valeur des poussées du terrain.

Lors de l'interprétation, l'examen de la ligne d'influence du front est également importante.

Deux types de critères sont à distinguer :

- les critères concernant la sécurité du chantier et la bonne mise en œuvre de la méthode de creusement ;

- les critères imposés par des travaux en site urbain. Ces derniers critères se surimposent aux précédents car ils sont généralement plus stricts.

\subsection{Critères intervenant pour la conduite du chantier}

Ces critères concernent essentiellement le comportement et la stabilité de la cavité et du massif encaissant et non pas les incidences possibles des travaux sur des ouvrages préexistants dans le voisinage. Ils 
s'appuient essentiellement sur des mesures de déplacement. Les valeurs admissibles sont évaluées en cherchant à atteindre un compromis entre les performances d'avancement et le prix de revient d'un côté et la sécurité de l'autre, en prenant des risques bien dosés.

Pour ce qui concerne l'utilisation de la nouvelle méthode autrichienne ou méthodes similaires, il n'existe pas encore de critères numériques admis et reconnus. Les valeurs admissibles dépendent, en effet, de nombreux facteurs (nature géotechnique du terrain et de son comportement, nature et géométrie de l'ouvrage, hauteur de couverture, etc.) sans considérer les problèmes de surface en site urbain. Il est très délicat d'avancer à ce sujet des critères quantitatifs. Nous nous limiterons à donner à titre purement indicatif et sous toute réserve, quelques valeurs numériques liées à notre propre expérience, en se plaçant dans le cas d'un ouvrage de section moyenne, c'est-à-dire comprise entre 50 et $100 \mathrm{~m}^{2}$. Ces critères correspondent au déplacement absolu de la clé de voûte (généralement de l'ordre de la moitié de la convergence). Seule une fourchette peut être donnée pour tenir compte des caractéristiques rhéologiques des terrains (terrains raides ou plastiques). Les différents facteurs intervenant dans la détermination des seuils admissibles sont successivement passés en revue :

\section{a) Amplitude des déplacements}

D'une manière générale, l'amplitude maximale admissible est essentiellement fonction de la hauteur de couverture. Elle est de l'ordre du millième de cette dernière. Le tableau ci-après nuance cette donnée générale selon la nature des terrains.

\begin{tabular}{|c|c|c|}
\hline \multirow{2}{*}{$\begin{array}{c}\text { Hauteur } \\
\text { de couverture }\end{array}$} & \multicolumn{2}{|c|}{$\begin{array}{c}\text { Déplacement absolu } \\
\text { en clé de voûte } \\
\text { maximum admissible }\end{array}$} \\
\cline { 2 - 3 } & $\begin{array}{c}\text { Terrains raides } \\
\text { Terrains } \\
\text { plastiques }\end{array}$ \\
\cline { 2 - 3 } 10 à $50 \mathrm{~m}$ & 6 à $12 \mathrm{~cm}$ & $\begin{array}{c}2 \text { à } 5 \mathrm{~cm} \\
10 \text { à } 20 \mathrm{~cm} \\
20 \text { à } 40 \mathrm{~cm}\end{array}$ \\
\hline 50 à $500 \mathrm{~m}$ & $\begin{array}{c}2 \text { à } 6 \mathrm{~cm} \\
>500 \mathrm{~m}\end{array}$ & 2 à \\
\hline
\end{tabular}

La détermination de maxima admissibles doit se faire cas par cas avec la plus grande prudence, en ayant soin de tenir compte du comportement des terrains (fragile, élasto-plastique, plastique).

\section{b) Vitesse de déplacement}

La vitesse de déplacement est généralement mesurée en $\mathrm{mm}$ par jour ou par poste. Elle est maximale au passage du front dans le plan de mesure. Le déplacement journalier admissible est de l'ordre du $1 / 5$ à $1 / 4$ du déplacement total admissible au passage du front et doit descendre en dessous du $1 / 20$ après un délai de l'ordre d'une semaine. La vitesse doit, enfin, s'annuler après une mise en œuvre complète du soutènement immédiat.

\section{c) Accélération du déplacement}

Les accélérations positives ne sont admissibles que dans les un ou deux jours qui précèdent ou suivent le passage du front (ouverture de la calotte ou abattage du stross) dans le plan de mesure. Au-delà de cet intervalle de temps, l'accélération doit toujours être négative et correspondre à un mouvement suffisam- ment décéléré, surtout si le revêtement définitif n'est pas prévu ou s'il est différé à long terme.

\section{d) Gradient de déplacement}

Le gradient des déplacements (ou des déformations) dans l'espace environnant le tunnel donne l'importance de la zone d'influence du creusement. L'idéal est d'obtenir une décroissance rapide des déplacements (gradient négatifs élevés) autour de la cavité.

Si les déplacements s'annulent totalement à l'intérieur de la zone boulonnée, une stabilité absolue est assurée. Malheureusement, il n'en est pas toujours ainsi, notamment dans les mauvais terrains et $a$ fortiori dans les terrains non boulonnables. Il conviendra d'être très vigilant dans de tels cas, notamment lorsqu'au moins $50 \%$ des déplacements maxima (obtenus généralement à la paroi de la cavité) débordent la zone boulonnée.

Dans le cas des ouvrages peu profonds, la situation devient préoccupante, notamment lorsque les tassements atteignent la surface sans atténuation.

L’importance des poussées du terrain peut être estimée à partir du volume de la zone d'influence du creusement.

Les critères présentés ici concernent le chantier, ils se rapportent donc à l'analyse de la stabilité à court terme. Le problème du comportement à long terme est beaucoup plus délicat, il y sera fait allusion en fin de ce chapitre.

\subsection{Critères liés aux travaux souterrains en site urbain}

Les difficultés supplémentaires liées aux travaux souterrains en site urbain, généralement à faible profondeur, résultent de l'incidence des travaux sur les ouvrages environnants, généralement situés en surface.

L'auscultation en site urbain devra donc être axée davantage sur l'observation et le contrôle des mouvements et désordres en surface (tassements, fissuration d'ouvrages, etc.). Elle conduit généralement à des tolérances beaucoup plus faibles que celles avancées précédemment.

Des règles générales n'ont, pour le moment, pas encore été formulées.

Les limites généralement admises portent sur :

- l'amplitude maximale de l'affaissement de l'ordre de 1 à $2 \mathrm{~cm}$ selon la nature et la qualité des constructions en surface ;

- la pente de la dépression au point d'inflexion qui doit rester inférieure à $1 / 300$.

Dans certains cas spéciaux, par exemple lors du passage en tunnel sous des ouvrages particulièrement vulnérables ou sensibles (voies de chemin de fer à grande circulation, conduite d'eau en charge, immeubles vétustes, etc.), des conditions particulières peuvent être imposées. A titre d'exemple, nous citerons le cas du passage délicat [du métro (S-Bahn)] de Bochum à quelques mètres sous des voies de grandes lignes de la Bundesbahn (Société des chemins de fer allemands), qui avait prescrit un tassement journalier maximal de $4 \mathrm{~mm}$ et une amplitude totale maximale (tassements cumulés) de $15 \mathrm{~mm}$. A l'exécution, ces prescriptions furent respectées malgré la grande section du tunnel $\left(s=70 \mathrm{~m}^{2}\right)$ et la qualité médiocre des terrains traversés (marnes gréseuses). Ce succès fut obtenu essentiellement grâce à une parfaite mise en œuvre de la nouvelle méthode autrichienne. 
